Originally published as:

Juliane Schroeder, Toni Aebischer.

Vaccines for leishmaniasis: From proteome to vaccine candidates.

(2011) Human Vaccines, 7 (Suppl.), pp. 10-15.

DOI: 10.4161/hv.7.0.14556

This is an author manuscript.

The definitive version is available at: http://www.landesbioscience.com 


\title{
Vaccines for leishmaniasis: From proteome to vaccine candidates
}

\author{
Juliane Schroeder $1^{\dagger}$, Toni Aebischer 1, 2* \\ ${ }^{1}$ Marie Curie Excellence Team Pathogen Habitats; Institute of Immunology and Infection Research; \\ University of Edinburgh; UK; 2Robert Koch Institute; Berlin, Germany \\ ${ }^{\dagger}$ Current address: Strathclyde Institute for Pharmacy and Biomedical Science; University of \\ Strathclyde; Glasgow, UK \\ *Correspondence to: Toni Aebischer; Email: AebischerA@rki.de
}

\begin{abstract}
Leishmania spp. cause a wide spectrum of tropical diseases which are threatening an estimated 350 million people around the globe. While in most cases non-fatal, the disease is associated with high morbidity, social stigmata and poverty.

However, the most severe form visceral leishmaniasis can be fatal if left untreated.

Chemotherapeutics are available but show high toxicity, costs and are prone to resistance development due to prolonged treatment periods. Healing is associated with a life-long resistance to re-infection and this argues for the feasibility of vaccination. However, despite much effort, no such vaccine has become available yet.

Here, the status of vaccine development in this field is briefly summarized before the focus is set on the promise of reverse vaccinology for anti-Leishmania vaccine development in the post-genomic era. We report on our own experience with this approach using an instructive example of successful candidate vaccine antigen identification.
\end{abstract}

\section{Leishmaniases-The Problem}

Leishmaniases-a group of infectious diseases caused by at least 20 species of insect-transmitted protozoan parasites of the genus Leishmania-belong to the most neglected diseases. The latter are illnesses of the poor not attracting enough political and financial support to engage in adequate research and development of effective measures to prevent or treat the condition.

The degree of neglect and the burden of leishmaniasis is difficult to analyze and the often cited numbers of 12 million patients worldwide amongst 350 million people at risk in 88 countries endemic for Leishmania spp. (www.who.int/leishmaniasis/en/) do not fully relate the impact. The methodology to estimate the scale of the problem is still crude1 due to the fact that leishmaniasis is not one disease but represents several syndromes.

These range from self-healing and chronic cutaneous lesions $(C L)$ to mucocutaneous $(M L)$ and visceral leishmaniasis (VL) which is often fatal. In addition, its epidemiology is complex and includes zoonotic and anthroponotic cycles (Fig. 1). Nonetheless, efforts to fathom the problem by relational approaches rank it in mortality second only to malaria and fourth in morbidity among all tropical parasitic diseases.2

Preventing people from dying from VL has therefore become a priority. Fatal disease is linked to visceral infection caused by $L$. donovani and $L$. infantum in the old world and additionally to L. chagasi in the New World. Over $90 \%$ of VL occurs in India and Sudan.

In India VL reaches annual incidence rates of 2.5/1,000 person in highly endemic areas and prevalence of $L$. donovani infection based on serological evidence is currently estimated at 
$18 \% .3$ Only few drugs are available for the foreseeable future to care for these patients. 4 In fear of the emergence of drug resistant parasites, combinatorial therapies are advocated but these remain costly and expense for the most cost effective combination to avert an otherwise fatal infection is estimated at US $\$ 80$ equivalents. 5 For comparison this corresponds to roughly 2.5 months of average income in the area most affected by VL in India. 6 Thus, the economic burden of treatment and knock on cost of VL represents $>10 \%$ of an average household's expenditure forcing many into spiraling poverty. 6

\section{Feasibility of Vaccination}

To develop a vaccine against leishmaniasis to complement disease management options has been a priority for some years. Given that many Leishmania species are involved and epidemiological scenarios vary greatly, it remains unclear whether one or many vaccines will be needed. Fact is, however, that no vaccine is currently available for protecting humans from VL, ML or CL. The mortality associated with VL caused by $L$. donovani argues for giving priority to develop a vaccine against VL.

The feasibility of a vaccine is extrapolated from the fact that self healing CL appears to confer life-long protection against the same disease. This has been exploited in the century old practice of leishmanization, i.e., the deliberate infection with parasites at body sites where ensuing scars will not be stigmatizing.7,8 The strategy was successfully used for mass prevention of $C L$ in particular by nations in the middle east but adverse effects and local lesions persisting for months in $2-3 \%$ of cases 9 clearly demand improvements and alternatives.

Thus, first generation vaccines based on crude preparations such as autoclaved in vitro grown parasites were developed to offer a safer alternative. Although protective in pre-clinical models they showed no efficacy after two decades of diverse clinical trials.

10 The experience with these vaccines highlights a critical issue in the comparatively resource poor programs of vaccine development against this disease: Low predictive power of available pre-clinical models and very slow and costly progress of prophylactic clinical vaccine testing programs. In recognition of these hurdles, improvements and alternative testing strategies have recently been proposed. For example, the predictive power of the pre-clinical mouse model to assess prophylactic vaccines may be improved when using an insect-vector challenge. 11 Leishmanization is advocated as a means to assess vaccine efficacy in clinical trials as a surrogate of natural infection.12 This will likely accelerate clinical development of prophylactic vaccines.

Similarly, protocols testing vaccination as an extended therapy have entered human trials.13 Again, this strategy will accelerate clinical developments since estimates of vaccine effects can be obtained faster and with fewer volunteers. The value of these approaches to develop 'resource conscious' strategies cannot be overestimated.

\section{Status of Anti-Leishmanial Vaccine Development}

The rational and status of vaccine development against leishmaniasis has been reviewed recently in references 14 and 15 .

Based on data from mouse models, a vaccine should induce cell mediated immunity, i.e., parasite antigen-specific, CD4+ and CD8+ T cells. Furthermore, it should induce immunological memory with appropriate tissue homing characteristics and effector function that activate leishmanicidal mechanisms in the major host cell types. During their life cycle all Leishmania oscillate between the insect transmitted extracellular promastigote and the intracellular amastigote form that thrives in host phagocytic cell types such as macrophages and dendritic cells (Fig. 1). Thus, a common perception is that a type 1 immune response characterized by so called poly-functional Tcells that produce IFNy and additional pro-inflammatory cytokines 16 is sufficient but this is indeed far from being clear. 
For example, in human VL, the determination of cytokine patterns from patients undergoing drug therapy only identified negative correlations, i.e., high levels of IL-10 being correlated with uncontrolled disease.17,18 Thus, the big questions in vaccine design are not yet solved: Which antigen(s) should one select and how should these be delivered? Moreover, with no convincing marker of protective immunity yet known, developing appropriate vaccine delivery strategies will have solely to rely on efficacy data from clinical trials.

\section{From Genomes to Vaccines}

With genome sequences and high coverage proteome data now available,19-22 the vaccine antigen discovery process was propelled into the era of reverse vaccinology.23,24

Highly parallel antigen discovery approaches such as the screening of gene-expression libraries have already been pursued before the genome was known.

They led to identification of $B$ and T-cell antigens recognized during Leishmania infections in mice (reviewed in refs. 25 and 26) and in patients.27-30 A fusion of three such antigens, LEISH-F1 (also known as Leish-111f), identified in this way is the only subunit vaccine that has since entered clinical trials.13,31,32 Genomic information and bioinformatic analysis tools allowed extending these approaches.

For example, proteins featuring tandem repeats were originally identified by screening expression libraries with patient sera28 and this approach has now been 'reversed' by screening protozoan genomes for genes encoding proteins with tandem repeats and verifying their antigenicity.33 However, there are very few studies published that used indeed such a reversed approach

starting from genome, proteome or transcriptome data to derive and test candidate vaccine antigens. A prerequisite of reverse vaccinology is that a set of criteria can be identified to design an algorithm for vaccine candidate selection or enrichment. Several studies34-37 provide support for selection based on protein expression in the amastigote form, relative protein abundance and subcellular localization. These criteria are usually combined with sequence conservation across the parasite species and lack of homology to vaccine target organism.

For selection purposes, the last three criteria can be addressed using bioinformatics tools to analyze information from genome and sequence data repositories such as Genbank. We have chosen a proteomic approach21,22 to provide a resource for the first two criteria. We reasoned that the sensitivity of the adopted proteomic approach is close to the sensitivity of the immune system to respond to a particular protein in complex mixtures such as whole parasites.

Thus, together with similar publicly available data sets20 the proteome data now provides a useful vaccine antigen selection platform representing $\sim 20 \%$ of the predicted proteome from promastigotes and amastigotes. A relatively high agreement between the proteomic datasets that were generated from $L$. donovani20 and $L$. mexicana21,22 further suggests that for many if not most of these proteins their relative abundance will be the same across parasite species. Furthermore, our bioinformatic analyses verified that protein abundance is correlated to bias in codon usage. This bias has been predicted to affect translation efficiency.38 Thus, in the absence of proteomic evidence for a particular protein or classes of proteins (e.g., most membrane proteins) biased codon usage in the respective gene combined with mRNA abundance data from transcriptome analyses 39,40 may serve as a surrogate measure for relative protein abundance.

We have since initiated a reverse vaccinology program and, using the above criteria, so far identified two novel vaccine candidates from $L$. donovani that showed promise in mouse models of VL and CL (Schroeder et al., in preparation). In this program, proteins positively evaluated in the mouse models will be further analyzed for the presence of putative T-cell epitopes that may be presented to CD8+ T cells in the context of human HLA alleles representative for a population living in a highly endemic area such as India.

The involvement of CD8+ T cells in resistance against visceral leishmaniasis has been demonstrated in several studies.41-44 TriTrypDB (Kinetoplastid Genomics Resource, tritrypdb. org/tritrypdb/) as part of GeneDB also offers identification of T cell in particular CD8+ T-cell epitopes in proteins. Epitopes in GeneDB are listed by the "Immune Epitope Database and Analysis Resource" (IEDB, www.immuneepitope.org/). 
This database searches for sequence similarities between the protein in question and experimentally identified and verified epitopes from various kinetoplastid antigens. Experimentally confirmed epitopes are listed with their respective sequence, source, method of identification, e.g., B-cell assay, MHC-binding assays etc., and reference.

Although the database thereby provides a summary of all experimentally identified epitopes, omitting time-consuming literature searches, it is only reporting verified epitopes.

This may be good for reasons of reliability but is not helpful when new antigens are analyzed.

Moreover, when using the epitope identification tool on TriTrypDB, only two epitopes were predicted in our example, one of which was misplaced in non-coding gene regions, while the other comprised only two amino acids.

The overall prediction confidence was classified as low (see below and Fig. 2). However, more than 30 programs predicting MHC binding peptides, i.e., potential epitopes, based on different algorithms are available on the internet. A comparative study45 assessed performance and reliability of MHC prediction programs using a set of model antigens. The study revealed that matrix based programs (e.g., BIMAS and SYFPEITHI) were outperformed by non-linear predictors like NetMHC which is based on artificial neural networks (ANN). Therefore we decided to analyze our antigen and KMP-11 for reference using NetMHC (reviewed in refs. 46 and 47; www.cbs.dtu.dk/services/NetMHC/).

Predictions of binding peptides take MHC polymorphisms into account and for a vaccine aimed to induce protective CD8+ T-cells in a target population, e.g. in India, relevant HLA types

have to be considered. The population of India is highly diverse with several thousand endogamous groups, 325 functioning languages and 25 scripts. 48 However, visceral leishmaniasis is endemic in only three states in the North of India, Bihar, where $90 \%$ of all cases of VL are reported, eastern Uttar Pradesh and West Bengal.

There the composition of ethnic populations seems to be better defined. Within the framework of the Indian Genome Variation Consortium it was established that the majority population of these states is Caucasian (Uttar Pradesh, Bihar and admixture in West Bengal) and Australoid (West Bengal) with mongoloid influence. This was further confirmed by a study involving Asian Indians of the Delhi area.

It was suggested that the Indian population is, although essentially Caucasoid, in reality a mixture of Caucasian and oriental haplotypes/alleles.49 Accordingly the most common HLA class I alleles in the relevant population will be $A^{\star} 02, A^{\star} 24, A^{*} 11, A^{\star} 33$ in the HLA-A locus and $B^{\star} 07, B^{*} 35, B^{\star} 40, B^{\star} 57$ and $B^{\star} 58$ in the $B$ locus. Thus, for the purpose of predicting vaccine antigen-derived HLA binding epitopes it was decided to restrict the analysis to these most common haplotypes. Of note, the most ubiquitous Caucasian allele $A^{*} 0201$ is absent in the Indian population and hence was replaced with the more frequent $A^{*} 0211$.

The result of the epitope prediction with NetMHC is shown in Figure 2. Analysis of KMP-11 (bottom half) generates a cluster of predicted binding peptides in the N-terminal region of the protein, with one classified as strong binder (black, threshold affinity $\leq 50 \mathrm{nM}$ ) starting at position 2 and a number of weakly binding peptides (grey, threshold affinity $\leq 500 \mathrm{nM}$ ) in the region 2-21. The C-terminus in comparison is a lot less represented with one strong binder starting at position 78 and a couple of weak binders.

This is coherent with experimental data, since all three experimentally identified clusters (aa 1-33, aa 50-60, aa 70-86) have been retrieved with NetMHC,50 which indicated clustered binding regions $\mathrm{N}$-terminal (1-30) and C-terminal (71-90).

Interestingly, the majority of peptides found, is predicted to bind to the selected HLA-A haplotypes, while the algorithm detected only one weak binder for the chosen HLA-B haplotypes. Based on the agreement with experimental data for KMP-11, it was assumed that a similar analysis using our novel antigen would be valid.

Epitope prediction for the same haplotypes showed a higher frequency of peptides with strong and weak binding ability both for HLA-A and HLA-B than for KMP-11. In addition several of these peptides did show no homologies to any peptide present in the predicted human proteome thus would even qualify them for focused epitope-based recombinant vaccines to activate CD8+ cells. Obviously, experimental verification of these is a future priority. 


\section{Vaccine Formulations}

Vaccine formulations will be decisive for the success of an immunization scheme.

The recombinant fusion protein vaccine LEISH-F has been formulated with MPL-A as an adjuvant for human trials.13 This type of vaccine is relatively expensive due to the still high cost to produce the recombinant protein and may be suboptimal to induce also CD8+ besides CD4+ T cells.

In preclinical models several alternative strategies have been pursued.

Vaccines based on recombinant DNA were often favored because they are rather easy to construct, can be produce quickly, are versatile and can be multiplexed.51-55 In addition vectored formulations that exploit recombinant vaccine carriers such as vaccinia16,56,57 or adenovirus 16,58 derivatives as well as recombinant bacteria, e.g., Salmonella59-62 have also been tested.

Virus-based vaccines may be particularly suited to induce CD8+ T cells.

Experience in other fields 63 and the results of several studies that explored experimental antiLeishmania vaccines $57,59,64,65$ suggest that prime boost combinations with heterologous vaccine formulations offer synergies that may become very important. We choose to refine the approach of using recombinant, live salmonella (see Fig. 3 for a process chart). The reasons for this were that salmonella are produced at industrial scale for both human and veterinary vaccine applications. The technology is relatively old and often no longer protected by patents, thus offering a high degree of freedom to operate. Salmonella are able to induce CD8+ as well as CD4+ T cells and induce T cells to home to visceral organs.

Depending on serovar, these carriers offer a rather broad host range that can be exploited to tackle zoonotic, as well as anthroponotic cycles. Most importantly, they can be produced extremely cheaply, stocked in lyophilized form and applied as an oral vaccine.

In summary, if only one vaccine is needed to combat the different forms of leishmaniasis caused by the many parasites then it seems that we will have to make a choice and should mainly think about how to devise a resource conscious clinical selection strategy.

However, if many vaccines are needed because the syndromes require tailored solutions, then we will need to think even harder about such a strategy.

\section{Acknowledgements}

This work was financially supported by the European Commission Marie Curie Excellence Grant EXT25435 to TAe. 


\section{References}

1. Bern $\mathrm{C}$, Maguire $\mathrm{JH}$, Alvar J. Complexities of assessing the disease burden attributable to leishmaniasis. PLoS Negl Trop Dis 2008; 2:313.

2. Mathers CD, Ezzati M, Lopez AD. Measuring the burden of neglected tropical diseases: the global burden of disease framework. PLoS Negl Trop Dis 2007; 1:114.

3. Singh SP, Picado A, Boelaert M, Gidwani K, Andersen EW, Ostyn B, et al. The epidemiology of Leishmania donovani infection in high transmission foci in India. Trop Med Int Health 2010; 15:12-20.

4. Murray HW, Berman JD, Davies CR, Saravia NG. Advances in leishmaniasis. Lancet 2005; $366: 1561-77$.

5. Meheus F, Balasegaram M, Olliaro P, Sundar S, Rijal S, Faiz AMd, et al. Cost-Effectiveness analysis of combination therapies for visceral leishmaniasis in the Indian subcontinent. PLoS Negl Trop Dis 2010; 4:818.

6. Sarnoff R, Desai J, Desjeux P, Mittal A, Topno R, Siddiqui NA, et al. The economic impact of visceral leishmaniasis on rural households in one endemic district of Bihar, India. Trop Med Int Health 2010; 15:42-9.

7. Berberian DA. Vaccination and immunity against oriental sore. Transactions of the Royal Society of Tropical Medicine and Hygiene 1939; 33:87.

8. Nadim A, Javadian E, Tahvildar-Bidruni G, Ghorbani M. Effectiveness of leishmanization in the control of cutaneous leishmaniasis. Bull Soc Pathol Exot Filiales 1983; 76:377-83.

9. Hosseini SM, Hatam GR, Ardehali S. Characterization of Leishmania isolated from unhealed lesions caused by leishmanization. East Mediterr Health J 2005; 11:240-3.

10. Noazin S, Khamesipour A, Moulton LH, Tanner M, Nasseri K, Modabber F, et al. Efficacy of killed wholeparasite vaccines in the prevention of leishmaniasis: a meta-analysis. Vaccine 2009; 27:474753.

11. Peters NC, Kimblin N, Secundino N, Kamhawi S, Lawyer P, Sacks DL. Vector transmission of leishmania abrogates vaccine-induced protective immunity. PLoS Pathog 2009; 5:1000484.

12. Khamesipour A, Dowlati Y, Asilian A, Hashemi-Fesharki R, Javadi A, Noazin S, et al.

Leishmanization: use of an old method for evaluation of candidate vaccines against leishmaniasis.

Vaccine 2005; 23:3642-8.

13. Nascimento E, Fernandes DF, Vieira EP, Campos-Neto A, Ashman JA, Alves FP, et al. A clinical trial to evaluate the safety and immunogenicity of the LEISH-F1 + MPL-SE vaccine when used in combination with meglumine antimoniate for the treatment of cutaneous leishmaniasis. Vaccine 2010; 28:6581-7.

14. de Oliveira $\mathrm{Cl}$, Nascimento IP, Barral $\mathrm{A}$, Soto $\mathrm{M}$, Barral-Netto $\mathrm{M}$. Challenges and perspectives in vaccination against leishmaniasis. Parasitol Int 2009; 58:319-24.

15. Kedzierski L. Leishmaniasis vaccine: Where are we today? J Glob Infect Dis 2010; 2:177-85.

16. Darrah PA, Patel DT, De Luca PM, Lindsay RW, Davey DF, Flynn BJ, et al. Multifunctional TH1 cells define a correlate of vaccine-mediated protection against Leishmania major. Nat Med 2007; 13:843-50.

17. Kumar R, Goto Y, Gidwani K, Cowgill KD, Sundar S, Reed SG. Evaluation of ex vivo human immune response against candidate antigens for a visceral leishmaniasis vaccine. Am J Trop Med Hyg 2010; 82:808-13. 
18. Sundar S, Reed SG, Sharma S, Mehrotra A, Murray HW. Circulating T helper 1 (Th1) cell- and Th2 cellassociated cytokines in Indian patients with visceral leishmaniasis. Am J Trop Med Hyg 1997; $56: 522-5$

19. Ivens AC, Peacock CS, Worthey EA, Murphy L, Aggarwal G, Berriman M, et al. The genome of the kinetoplastid parasite, Leishmania major. Science 2005; 309:436-42.

20. Rosenzweig D, Smith D, Opperdoes F, Stern S, Olafson RW, Zilberstein D. Retooling Leishmania metabolism: from sand fly gut to human macrophage. FASEB J 2008; 22:590-602.

21. Paape D, Lippuner C, Schmid M, Ackermann R, Barrios-Llerena ME, Zimny-Arndt U, et al. Transgenic, fluorescent Leishmania mexicana allow direct analysis of the proteome of intracellular amastigotes. Mol Cell Proteomics 2008; 7:1688-701.

22. Paape D, Barrios-Llerena ME, Le BT, Mackay L, Aebischer T. Gel free analysis of the proteome of intracellular Leishmania mexicana. Mol Biochem Parasitol 2010; 169:108-14.

23. Rappuoli R. Reverse vaccinology. Curr Opin Microbiol 2000; 3:445-50.

24. Dumonteil E. Vaccine development against Trypanosoma cruzi and Leishmania species in the post-genomic era. Infect Genet Evol 2009; 9:1075-82.

25. Mougneau E, Altare F, Wakil AE, Zheng S, Coppola T, Wang ZE, et al. Expression cloning of a protective Leishmania antigen. Science 1995; 268:563-6.

26. Campos-Neto A, Soong L, Cordova JL, Sant'Angelo D, Skeiky YA, Ruddle NH, et al. Cloning and expression of a Leishmania donovani gene instructed by a peptide isolated from major histocompatibility complex class II molecules of infected macrophages. J Exp Med 1995; 182:1423-33.

27. Skeiky YA, Kennedy M, Kaufman D, Borges MM, Guderian JA, Scholler JK, et al. LelF: a recombinant Leishmania protein that induces an IL-12-mediated Th1 cytokine profile. J Immunol 1998; $161: 6171-9$.

28. Goto Y, Coler RN, Guderian J, Mohamath R, Reed SG. Cloning, characterization and serodiagnostic evaluation of Leishmania infantum tandem repeat proteins.

Infect Immun 2006; 74:3939-45.

29. Skeiky YA, Guderian JA, Benson DR, Bacelar O, Carvalho EM, Kubin M, et al. A recombinant Leishmania antigen that stimulates human peripheral blood mononuclear cells to express a Th1-type cytokine profile and to produce interleukin 12. J Exp Med 1995; 181:1527-37.

30. Probst P, Stromberg E, Ghalib HW, Mozel M, Badaro R, Reed SG, et al. Identification and characterization of T cell-stimulating antigens from Leishmania by CD4 T cell expression cloning. $J$ Immunol 2001; 166:498-505.

31. Coler RN, Goto Y, Bogatzki L, Raman V, Reed SG. Leish-111f, a recombinant polyprotein vaccine that protects against visceral leishmaniasis by elicitation of CD4+ T cells. Infect Immun 2007; 75:464854.

32. Llanos-Cuentas A, Calderon W, Cruz M, Ashman JA, Alves FP, Coler RN, et al. A clinical trial to evaluate the safety and immunogenicity of the LEISH-F1+MPL-SE vaccine when used in combination with sodium stibogluconate for the treatment of mucosal leishmaniasis. Vaccine 2010; 28:7427-35.

33. Goto Y, Coler RN, Reed SG. Bioinformatic identification of tandem repeat antigens of the Leishmania donovani complex. Infect Immun 2007; 75:846-51.

34. Aebischer T, Wolfram M, Patzer SI, Ilg T, Wiese M, Overath P. Subunit vaccination of mice against new world cutaneous leishmaniasis: comparison of three proteins expressed in amastigotes and six adjuvants. Infect Immun 2000; 68:1328-36. 
35. Wolfram M, Fuchs M, Wiese M, Stierhof YD, Overath P. Antigen presentation by Leishmania mexicana-infected macrophages: activation of helper $\mathrm{T}$ cells by a model parasite antigen secreted into the parasitophorous vacuole or expressed on the amastigote surface. Eur $\mathrm{J}$ Immunol 1996; 26:3153-62.

36. Prickett S, Gray PM, Colpitts SL, Scott P, Kaye PM, Smith DF. In vivo recognition of ovalbumin expressed by transgenic Leishmania is determined by its subcellular localization. J Immunol 2006; 176:4826-33.

37. Overath Pand Aebischer T. Antigen presentation by macrophages harboring intravesicular pathogens. Parasitol Today 1999; 15:325-32.

38. Horn D. Codon usage suggests that translational selection has a major impact on protein expression in trypanosomatids. BMC Genomics 2008; 9:2.

39. Holzer TR, McMaster WR, Forney JD. Expression profiling by whole-genome interspecies microarray hybridization reveals differential gene expression in procyclic promastigotes, lesion-derived amastigotes and axenic amastigotes in Leishmania mexicana. Mol Biochem Parasitol 2006; 146:198218.

40. Leifso K, Cohen-Freue G, Dogra N, Murray A, McMaster WR. Genomic and proteomic expression analysis of Leishmania promastigote and amastigote life stages: the Leishmania genome is constitutively expressed. Mol Biochem Parasitol 2007; 152:35-46.

41. Polley R, Stager S, Prickett S, Maroof A, Zubairi S, Smith DF, et al. Adoptive immunotherapy against experimental visceral leishmaniasis with CD8+ T cells requires the presence of cognate antigen. Infect Immun 2006; 74:773-6.

42. Stager S, Alexander J, Kirby AC, Botto M, Rooijen NV, Smith DF, et al. Natural antibodies and complement are endogenous adjuvants for vaccine-induced CD8+ T-cell responses. Nat Med 2003; 9:1287-92.

43. Basu R, Bhaumik S, Haldar AK, Naskar K, De T, Dana SK, et al. Hybrid cell vaccination resolves Leishmania donovani infection by eliciting a strong CD8+ cytotoxic T-lymphocyte response with concomitant suppression of interleukin-10 (IL-10) but not IL-4 or IL-13. Infect Immun 2007; 75:595666.

44. Mary C, Auriault V, Faugere B, Dessein AJ. Control of Leishmania infantum infection is associated with CD8(+) and gamma interferon- and interleukin-5-producing CD4(+) antigen-specific T cells. Infect Immun 1999; 67:5559-66.

45. Lin HH, Zhang GL, Tongchusak S, Reinherz EL, Brusic V. Evaluation of MHC-II peptide binding prediction servers: applications for vaccine research. BMC. Bioinformatics 2008; 9:22.

46. Buus S, Lauemoller SL, Worning P, Kesmir C, Frimurer T, Corbet S, et al. Sensitive quantitative predictions of peptide-MHC binding by a 'Query by Committee' artificial neural network approach. Tissue Antigens 2003; 62:378-84.

47. Nielsen M, Lundegaard C, Worning P, Lauemoller SL, Lamberth K, Buus S, et al. Reliable prediction of T-cell epitopes using neural networks with novel sequence representations. Protein Sci 2003; 12:1007-17.

48. Italian Genome Variation Consortium. The Indian Genome Variation database (IGVdb): a project overview. Hum Genet 2005; 118:1-11.

49. Mehra NK, Jaini R, Rajalingam R, Balamurugan A, Kaur G. Molecular diversity of HLA-A*02 in Asian Indians: predominance of $A^{\star} 0211$. Tissue Antigens 2001; 57:502-7.

50. Basu R, Roy S, Walden P. HLA class I-restricted T cell epitopes of the kinetoplastid membrane protein-11 presented by Leishmania donovani-infected human macrophages. J Infect Dis 2007; 195:1373-80. 
51. Ghosh A, Labrecque S, Matlashewski G. Protection against Leishmania donovani infection by DNA vaccination: increased DNA vaccination efficiency through inhibiting the cellular p53 response. Vaccine $2001 ; 19: 3169-78$.

52. Lopez-Fuertes L, Perez-Jimenez E, Vila-Coro AJ, Sack F, Moreno S, Konig SA, et al. DNA vaccination with linear minimalistic (MIDGE) vectors confers protection against Leishmania major infection in mice. Vaccine 2002; 21:247-57.

53. Walker PS, Scharton-Kersten T, Rowton ED, Hengge U, Bouloc A, Udey MC, et al. Genetic immunization with glycoprotein 63 cDNA results in a helper T cell type 1 immune response and protection in a murine model of leishmaniasis. Hum Gene Ther 1998; 9:1899-907.

54. Almeida R, Norrish A, Levick M, Vetrie D, Freeman T, Vilp J, et al. From genomes to vaccines: Leishmania as a model. Philos Trans R Soc Lond Biol Sci 2002; 357:5-11.

55. Stober CB, Lange UG, Roberts MT, Gilmartin B, Francis R, Almeida R, et al. From genome to vaccines for leishmaniasis: screening 100 novel vaccine candidates against murine Leishmania major infection. Vaccine 2006; 24:2602-16

56. Stober CB, Lange UG, Roberts MT, Alcami A, Blackwell JM. Heterologous priming-boosting with DNA and modified vaccinia virus Ankara expressing tryparedoxin peroxidase promotes long-term memory against Leishmania major in susceptible BALB/c Mice. Infect Immun 2007; 75:852-60.

57. Perez-Jimenez E, Kochan G, Gherardi MM, Esteban M. MVA-LACK as a safe and efficient vector for vaccination against leishmaniasis. Microbes Infect 2006; 8:810-22.

58. Gabaglia CR, Pedersen B, Hitt M, Burdin N, Sercarz EE, Graham FL, et al. A single intramuscular injection with an adenovirus-expressing IL-12 protects BALB/c mice against Leishmania major infection, while treatment with an IL-4-expressing vector increases disease susceptibility in B10.D2 mice. J Immunol 1999; 162:753-60.

59. Lange UG, Mastroeni P, Blackwell JM, Stober CB. DNA-Salmonella enterica serovar Typhimurium primerbooster vaccination biases towards $T$ helper 1 responses and enhances protection against Leishmania major infection in mice. Infect Immun 2004; 72:4924-8.

60. McSorley SJ, Xu D, Liew FY. Vaccine efficacy of Salmonella strains expressing glycoprotein 63 with different promoters. Infect Immun 1997; 65:171-8.

61. Xu D, McSorley SJ, Chatfield SN, Dougan G, Liew FY. Protection against Leishmania major infection in genetically susceptible BALB/c mice by gp63 delivered orally in attenuated Salmonella typhimurium (AroAAroD-). Immunology 1995; 85:1-7.

62. Yang DM, Fairweather N, Button LL, McMaster WR, Kahl LP, Liew FY. Oral Salmonella typhimurium (AroA-) vaccine expressing a major leishmanial surface protein (gp63) preferentially induces T helper 1 cells and protective immunity against leishmaniasis. J Immunol 1990; 145:2281-5.

63. Hill AV, Reyes-Sandoval A, O'Hara G, Ewer K, Lawrie A, Goodman A, et al. Prime-boost vectored malaria vaccines: progress and prospects. Hum Vaccin 2010; 6:78-83.

64. Dondji B, Perez-Jimenez E, Goldsmith-Pestana K, Esteban M, Mahon-Pratt D. Heterologous prime-boost vaccination with the LACK antigen protects against murine visceral leishmaniasis. Infect Immun 2005; 73:5286-9.

65. Ramos I, Alonso A, Marcen JM, Peris A, Castillo JA, Colmenares M, et al. Heterologous primeboost vaccination with a non-replicative vaccinia recombinant vector expressing LACK confers protection against canine visceral leishmaniasis with a predominant Th1-specific immune response. Vaccine 2008; 26:333-44. 


\section{Figures}

Figure 1. The life cycles of Leishmania spp. Flagellated promastigotes are transmitted by bloodsucking phlebotomines (Photo cWHO/ TDR) to vertebrate hosts where they are taken up by phagocytes and multiply intracellularly causing disease. The epidemiology of VL knows anthroponotic and zoonotic (Photo c Wellcome Trust IHC) cycles.

Definitive diagnosis of VL is obtained by parasite detection in visceral organ biopsies (e.g., splenic probing; photo courtesy of J. Clos).

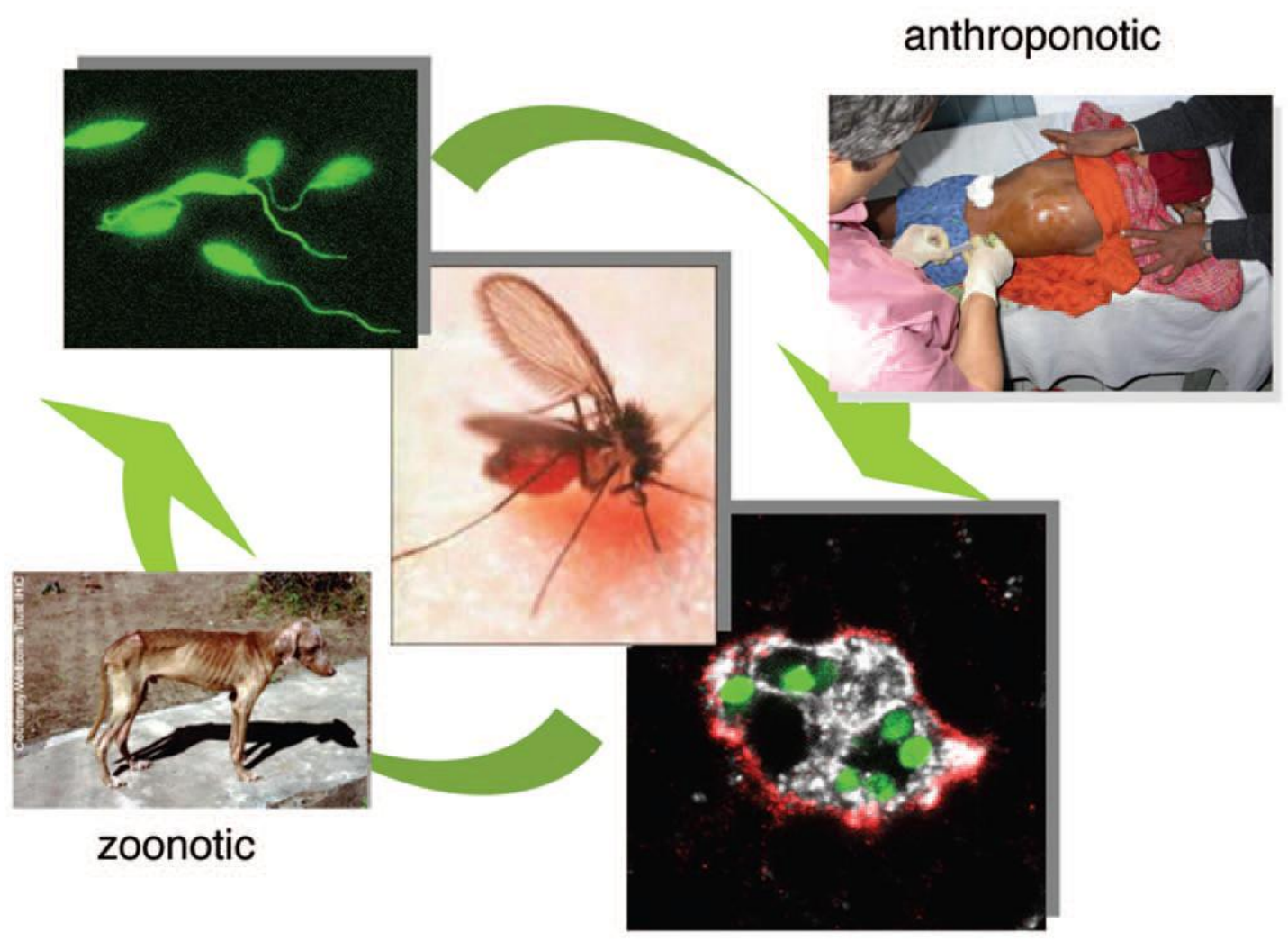


Figure 2. MHC class I epitope prediction for novel L. donovani antigen L_08 and KMP-11. NetMHC (www.cbs.dtu.dk/services/NetMHC/) was used on a selected set of HLA-A and B haplotypes/alleles. Immunogenic peptides are depicted with a bar symbolizing a nonamer. Weak binders (threshold affinity $\leq 500 \mathrm{nM}$ ) are shown in grey, strong binders (threshold affinity $\leq 50 \mathrm{nM}$ ) are shown in black and the position of the only L_08 di-peptide epitope predicted by TriTrypDB within the ORF is indicated by bigger font numbers.

Novel L. donovani antigen L_08

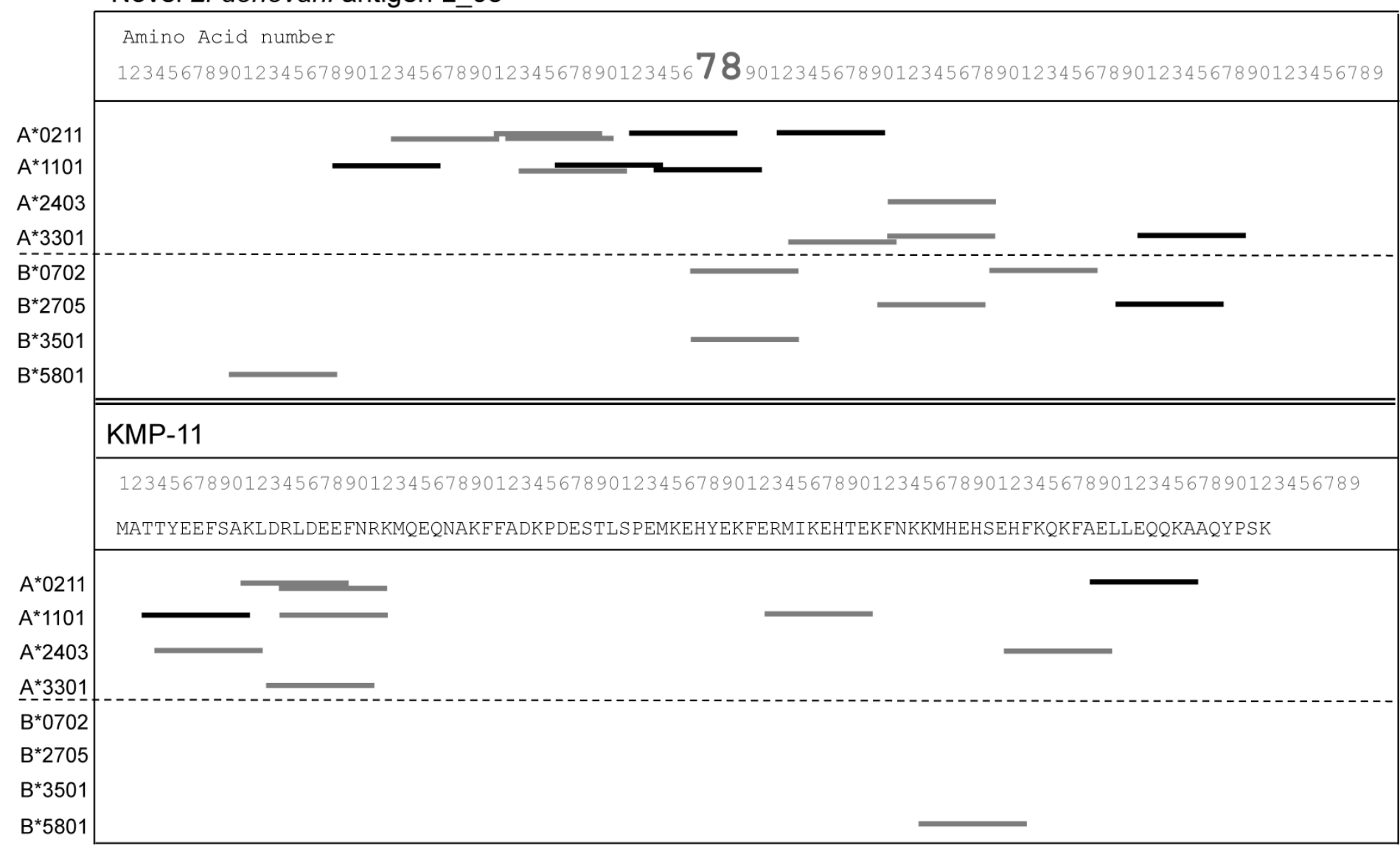


Figure 3. Cloning and pre-clinical selection strategy for antigen and vaccine candidates. Antigens have been selected from a proteomic dataset based on bioinformatic criteria, cloned into surface and cytosolic expression systems. Carrier salmonella expressing vaccine candidates are being tested for bacterial fitness in vivo and antigen expression to select optimal carrier strains that are subsequently evaluated in different mouse Leishmania infection models. Antigens conferring protection in this format are then further characterized for the presence human HLA class I epitopes.

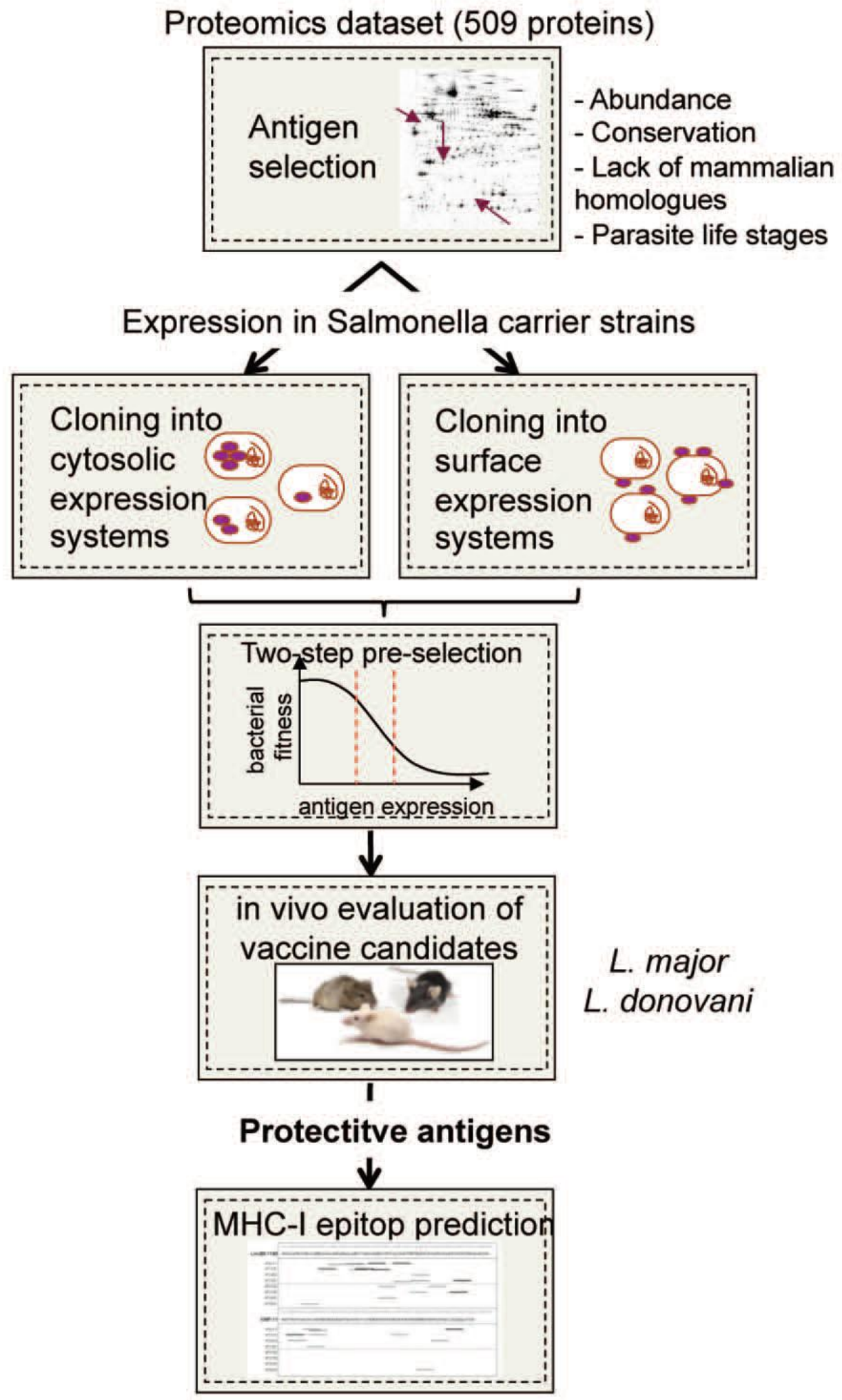

